\title{
Anti-inflammatory effects of methanol extract of Canarium lyi C.D. Dai \& Yakovlev in RAW 264.7 macrophages and a murine model of lipopolysaccharide-induced lung injury
}

\author{
JU-MI HONG ${ }^{1,2}$, OK-KYOUNG KWON ${ }^{1}$, IN-SIK SHIN ${ }^{1}$, CHAN-MI JEON $^{1}$, \\ NA-RAE SHIN ${ }^{1}$, JOONGKU LEE ${ }^{3}$, SANG-HONG PARK $^{3}$, TRAN THE BACH $^{4}$, DO VAN HAI ${ }^{4}$, \\ SEI-RYANG $\mathrm{OH}^{1}$, SANG-BAE HAN ${ }^{2}$ and KYUNG-SEOP AHN ${ }^{1}$
}

\begin{abstract}
${ }^{1}$ Natural Medicine Research Center, Korea Research Institute of Bioscience and Biotechnology, Chungbuk 363-883;
${ }^{2}$ College of Pharmacy, Chungbuk National University, Cheongju, Chungbuk 361-763; ${ }^{3}$ International Biological Material Research Center, Korea Research Institute of Bioscience and Biotechnology, Daejeon 305-806, Republic of Korea;

${ }^{4}$ IEBR, Vietnam Academy of Science and Technology (VAST), Cau Giay, Ha Noi 10307, Vietnam
\end{abstract}

Received June 9, 2014; Accepted February 18, 2015

DOI: $10.3892 / \mathrm{ijmm} .2015 .2117$

\begin{abstract}
Canarium lyi C.D. Dai \& Yakovlev (CL) is a member of the Anacardiaceae family. To the best of our knowledge, no studies on its anti-inflammatory effects have yet been reported. In the present study, we investigated the protective effects of CL on inflammation in lipopolysaccharide (LPS)-stimulated RAW 264.7 cells and LPS-induced acute lung injury (ALI) mice. CL attenuated the production of LPS-stimulated inflammatory mediators such as tumor necrosis factor- $\alpha$ (TNF- $\alpha$ ), interleukin (IL)-1 $\beta$, and interleukin-6 (IL-6). Furthermore, CL suppressed phosphorylation of the inhibitor $\kappa \mathrm{B}-\alpha(\mathrm{I} \kappa \mathrm{B}-\alpha)$, p38, c-Jun terminal kinase (JNK) and extracellular signal-regulated kinase (ERK), as well as the translocation of the nuclear factor $-\kappa \mathrm{B}(\mathrm{NF}-\kappa \mathrm{B}) \mathrm{p} 65$ subunit into the nucleus. For the in vivo efficacy, the effect of CL on a mouse model of LPS-induced acute lung injury was assessed. CL treatment of the mice significantly inhibited the inflammatory cell recruitment and pro-inflammatory cytokine production in bronchoalveolar lavage fluids (BALF). CL-treated mice also showed a marked inhibition of cyclooxygenase-2 (COX-2) and phosphorylation of $\mathrm{I} \kappa \mathrm{B}$ and $\mathrm{p} 65$. In addition, CL attenuated lung histopathological changes in LPS-induced ALI mice. In conclusion, our
\end{abstract}

Correspondence to: Dr Kyung-Seop Ahn, Natural Medicine Research Center, Korea Research Institute of Bioscience and Biotechnology, 30 Yeongudanji-ro, Ochang-eup, Cheongwon-gu, Cheongju-si, Cheongwon-gun, Chungbuk 363-883, Republic of Korea E-mail:ksahn@kribb.re.kr

Professor Sang-Bae Han, College of Pharmacy, Chungbuk National University, 52 Naesudong-ro, Heungdeokgu, Cheongju-si, Chungbuk 361-763, Republic of Korea

E-mail: shan@chungbuk.ac.kr

Key words: Canarium lyi C.D. Dai \& Yakovlev, inflammation, lipopolysaccharide, acute lung injury results suggest that $\mathrm{CL}$ is a potential therapeutic candidate for the treatment of inflammatory diseases, including pneumonia.

\section{Introduction}

Inflammation is a complex protective response caused by endoand exogenous stimuli such as bacterial lipopolysaccharide (LPS) (1). LPS, the major component in the outer membrane of gram-negative bacteria cell walls, induces the production of pro-inflammatory cytokines, such as tumor necrosis factor- $\alpha$ (TNF- $\alpha$ ), interleukin (IL)-1 $\beta$, and IL-6, as well as inflammatory mediators, such as nitric oxide (NO) and prostaglandin $\mathrm{E}_{2}\left(\mathrm{PGE}_{2}\right)$, which are synthesized by inducible nitric oxide synthase (iNOS) and cyclooxygenase-2 (COX-2), respectively (2). These pro-inflammatory cytokines and inflammatory mediators assist with the innate immune response. However, their overproduction results in acute phase endotoxemia that causes tissue injury, organ failure, shock and even death (3).

Nuclear factor- $\kappa \mathrm{B}(\mathrm{NF}-\kappa \mathrm{B})$, which is a key player in the regulation of immune and inflammatory responses, has an important role in modulating the transcription of several inflammatory factors and cytokines such as TNF- $\alpha$, IL-1 $\beta$ and IL-6 (4). In unstimulated cells, Rel protein dimers, which are composed mainly of p50 and p65 subunits, are normally sequestered in the cytosol as an inactive complex by binding to inhibitor $\kappa \mathrm{B}-\alpha(\mathrm{I} \kappa \mathrm{B}-\alpha)(5)$. The activation of NF- $\kappa \mathrm{B}$ mostly occurs through the phosphorylation and subsequent degradation of $\mathrm{I} \kappa \mathrm{B}$ via the activation of inhibitor $\kappa \mathrm{B}$ kinase (IKK). When $\mathrm{I} \kappa \mathrm{B}$ is phosphorylated, it is targeted for ubiquitination and subsequent degradation by the $26 \mathrm{~S}$ proteosome (6). The resulting free $\mathrm{NF}-\kappa \mathrm{B}$ then translocates to the nucleus, where it binds to $\kappa \mathrm{B}$-binding sites in the promoter regions of target genes and induces the transcription of pro-inflammatory mediators (7).

The mitogen-activated protein kinases (MAPKs) are serine/threonine-specific protein kinases that play a critical role in the regulation and differentiation of cell survival/apoptosis, as well as in controlling the cell response to cytokines, 
growth factors and environmental stresses (8). These classical MAPKs, extracellular signal-regulated kinase 1/2 (ERK 1/2), p38 MAPK and c-jun NH2-terminal kinase (JNK) have been involved in the transcriptional regulation of inflammatory genes (9). Specifically, p38 MAPK signaling pathways constitute an additional level of gene regulation by $\mathrm{NF}-\kappa \mathrm{B}$, especially of the p65 subunit $(10,11)$. Synergistically, MAPKs and NF- $\mathrm{BB}$ can collaborate to induce pro-inflammatory cytokine gene products and excretion (12). Accordingly, therapy aimed at the inhibition of NF- $\kappa \mathrm{B}$ and MAPKs have potential therapeutic advantages in curing inflammatory diseases (13).

Canarium lyi C.D. Dai \& Yakovlev (CL) is a member of the Anacardiaceae family. To the best of our knowledge, no studies on its anti-inflammatory effects have yet been reported.

In the present study, we elucidated the assumptive antiinflammatory mechanism of CL on LPS-stimulated RAW 264.7 macrophages by measuring the levels of cytokines, $\mathrm{NF}-\kappa \mathrm{B}$ and MAPK activation. In addition, we assessed the antiinflammatory effects of CL in a murine model of LPS-induced acute lung injury by measuring the brochoalveolar lavage fluid (BALF) analysis, pro-inflammatory protein expression and histological alteration of lung tissue.

\section{Materials and methods}

\section{In vitro experiment}

Preparation of plant material. Canarium lyi C.D. Dai \& Yakovlev of the Burseraceae family was collected from the area of Gia Lai, K Bang, So Pai, Vietnam in 2011. Plant samples were identified by Dr Tran The Bach of the Institute of Ecology and Biological Resources. A voucher specimen (KRIB 0036679) was deposited in the herbarium (KRIB) of the Korea Research Institute of Bioscience and Biotechnology. Canarium lyi (147 g) was treated with $\mathrm{MeOH}$ and sonicated several times at room temperature for 3 days to produce an extract $(20.05 \mathrm{~g})$.

Cell culture. RAW 264.7 cells were maintained at $1 \times 10^{5}$ cells/ml in Dulbecco's modified Eagle's medium (DMEM; Sigma, St. Louis, MO, USA) supplemented with $10 \%$ heatinactivated fetal bovine serum (FBS; Invitrogen, Burlington, ON, Canada), and $1 \%$ (w/v) of an antibiotic-antimycotic solution (Invitrogen, Grand Island, NY, USA) in 95\% air and 5\% $\mathrm{CO}_{2}$ humidified atmosphere at $37^{\circ} \mathrm{C}$.

Cytotoxicity assay. Cell viability was determined by assessing the mitochondrion-dependent reduction of MTT (Amresco LLC, Solon, OH, USA) to formazan. Briefly, $5 \mu 1$ of a $5 \mathrm{mg} / \mathrm{ml}$ MTT solution was added to the cell supernatant, and then incubation for $4 \mathrm{~h}$ at $37^{\circ} \mathrm{C}$. DMSO was added following removal of the medium. The optical density of formazan was measured using a microplate reader (VersaMax; Molecular Devices, San Diego, CA, USA) at $570 \mathrm{~nm}$. The level of formazan generated by untreated cells was chosen as the $100 \%$ value.

Measurement of nitric oxide. Nitrite levels in the cultured media and serum, which reflect intracellular nitric oxide (NO) synthase activity, were determined by Griess reaction. The cells were incubated with samples in the presence of LPS $(0.5 \mu \mathrm{g} /$ $\mathrm{ml}$, Sigma-Aldrich, La Jolla, CA, USA) at $37^{\circ} \mathrm{C}$ for $24 \mathrm{~h}$. The cell supernatant was dispensed into new 96-well plates, and $100 \mu \mathrm{l}$ of each supernatant was mixed with the same volume of the Griess reagent [1\% sulfanilamide, $0.1 \% \mathrm{~N}$-(1-naphathyl)ethylenediamine dihydrochloride and $5 \%$ phosphoric acid] and incubated at room temperature for $10 \mathrm{~min}$. Sodium nitrite was used to generate a standard curve, and the concentration of nitrite was measured for absorbance at $540 \mathrm{~nm}$.

Enzyme-linked immunosorbent assay (ELISA) of IL-6. The levels of IL-6 in supernatant were determined using a commercially available ELISA kit (R\&D Systems Inc., Minneapolis, MN, USA) according to the manufacturer's instructions. IL-6 levels were determined from a standard curve. The concentrations were expressed as $\mathrm{pg} / \mathrm{ml}$.

Enzyme immune assay of prostaglandin $E_{2}$. PGE $\mathrm{PG}_{2}$ levels in supernatants were determined using a $\mathrm{PGE}_{2}$ EIA kit (Cayman Chemical Co., Inc., Ann Arbor, MI, USA) according to the manufacturer's instructions. Briefly, $50 \mu \mathrm{l}$ diluted standards/samples were pipetted into the wells of a 96-well plate precoated with goat polyclonal anti-mouse IgG. Aliquots of a $\mathrm{PGE}_{2}$ monoclonal antibody solution and a $\mathrm{PGE}_{2}$ acetylcholine esterase conjugate solution were added to each well, and incubated at room temperature for $18 \mathrm{~h}$. The wells were washed six times with a wash buffer containing $0.05 \%$ (v/v) Tween-20, followed by the addition of $200 \mu \mathrm{l}$ Ellman's reagent containing acetylthiocholine and 5,5'-dithio-bis-(2-nitrobenzoic acid). $\mathrm{PGE}_{2}$ concentrations were measured by absorbance at $405 \mathrm{~nm}$.

Reverse transcriptase-polymerase chain reaction analysis $(R T-P C R)$. Total RNA was isolated using TRIzol ${ }^{\mathrm{TM}}$ reagent (Life Technologies Corp., Carlsbad, CA, USA). For RT-PCR, a single-strand cDNA was synthesized from $2 \mu \mathrm{g}$ total RNA. The primer sequences used were: iNOS: sense, 5'-CAA GAG TTT GAC CAG AGG ACC-3' and antisense, 5'-TGG AAC CAC TCG TAC TTG GGA-3'; COX-2: sense, 5'-GAA GTC TTT GGT CTG GTC TCC TG-3' and antisense, 5'-GTC TGC TGG TTT GGA ATA GTT GC-3'; TNF- $\alpha$ : sense and 5'-CAT CTT GCA AAA TTC GAG TGA CAA-3' and antisense, 5'-TGG GAG TAG ACA AGG TAC AAC CC-3'; IL-6, sense, 5'-GAG GAT ACC ACT CCC AAC AGA CC-3' and antisense, 5'-AAG TGC ATC ATC GTT GTT CAT ACA-3', $\beta$-actin: sense, 5'-CGC TCA TTG CCG ATA GTG AT-3' and antisense 5'-TGT TTG AGA CCT TCA ACA CC-3'. PCR products were fractionated on $1.5 \%$ agarose gel electrophoresis and stained with $5 \mu \mathrm{g} / \mathrm{ml}$ ethidium bromide. Images were captured by an Olympus C-4000 Zoom camera system (Olympus America Inc., Center Valley, PA, USA).

Immunoblot analysis. Western blot analyses were performed as previously described (14). Immunoblotting was performed with the primary antibodies at $4^{\circ} \mathrm{C}$ overnight. A horseradish peroxidase-labeled secondary antibody (Santa Cruz Biotechnology Inc., Santa Cruz, CA, USA) was then used for $1 \mathrm{~h}$. The membranes were washed three times with TBST, and then developed using an enhanced chemiluminescence (ECL) kit (Thermo Fisher Scientific, San Jose, CA, USA). For quantitative analysis, densitometric band values were determined using a bio-imaging analyzer (LAS 4000 mini; Fujifilm, Tokyo, Japan). 
In vivo experiment

Animals. Male C57BL/6 mice (6-8 weeks) were obtained from the Koatech Co. (Pyeongtaek, Korea). The mice were fed with food and water ad libitum in an animal facility with temperature ranging from 22 to $24^{\circ} \mathrm{C}$ and a 12-h light/ dark cycle under a specific pathogen-free conditions. Prior to the initiation of the experiment, the mice were housed for a minimum of one week in order that they adapt to the environment. The animal experimental procedures were approved by the Korea Research Institute of Bioscience and Biotechnology and performed in compliance with the National Institute of Health Guidelines for the care and use of laboratory animals and the Korean national animal welfare law.

Experimental protocols. Mice were randomly allocated into 4 groups: Control, LPS, LPS + dexamethasone (LPS + DEX) and LPS + CL. Dexamethasone served as a positive control drug. The mice of the LPS + DEX and LPS + CL groups received dexamethasone $(3 \mathrm{mg} / \mathrm{kg})$ and $\mathrm{CL}(30 \mathrm{mg} / \mathrm{kg})$ by oral gavage for 3 days, respectively. The mice from the control and LPS groups received oral gavage at an equal volume of PBS. The mice including the LPS, LPS + DEX and LPS + CL groups were instilled with $10 \mu \mathrm{g}$ of LPS dissolved in $50 \mu 1 \mathrm{PBS}$ intranasally to induce acute lung injury $1 \mathrm{~h}$ after final drug treatment. Mice in the control group were intranasally given $50 \mu 1$ PBS without LPS for $18 \mathrm{~h}$. To obtain BALF, ice-cold PBS $(0.7 \mathrm{ml})$ was infused into the lung and withdrawn via tracheal cannulation twice (total volume $1.4 \mathrm{ml}$ ).

Inflammatory cell counts in bronchoalveolar lavage fluid. Total inflammatory cell numbers were assessed by counting cells in at least five squares of a hemocytometer after excluding dead cells by Trypan blue staining. To determine the differential cell counts, $100 \mu 1$ of BALF was centrifuged onto slides using a Cytospin (Hanil Science Industrial Co., Ltd., Seoul, Korea) (200 x g for $5 \mathrm{~min}$ ). The slides were dried, and the cells were fixed and stained using a Diff-Quik ${ }^{\circledR}$ staining reagent (B4132-1A; IMEB Inc., Deerfield, IL, USA) following the manufacturer's instructions. The supernatant obtained from BALF was stored at $-70^{\circ} \mathrm{C}$ for the biochemical analysis.

Western blot analysis of the lung tissue. Lung tissue was homogenized $(1 / 10 \mathrm{w} / \mathrm{v})$ using a homogenizer with a tissue lysis/extraction reagent (Sigma-Aldrich) containing a protease inhibitor cocktail (Roche Diagnostics, Indianapolis, IN, USA). Each protein concentration was determined using a Bradford reagent (Bio-Rad Laboratories, Hercules, CA, USA). Western blotting was performed as described above, and the levels of $\mathrm{COX}-2, \mathrm{I} \kappa \mathrm{B}-\alpha$ and $\mathrm{NF}-\kappa \mathrm{B}$ were determined.

Measurement of the levels of pro-inflammatory cytokines in the BALF. The levels of IL-6 (R\&D Systems), TNF- $\alpha$ and IL- $1 \beta$ (BD Biosciences, San Jose, CA, USA) in BALF were measured using ELISA kits according to the manufacturer's instructions.

Histological analysis. After BALF samples were obtained, lung tissues were fixed in $4 \%(\mathrm{v} / \mathrm{v})$ paraformaldehyde. Tissues were embedded in paraffin, sectioned at $4 \mu \mathrm{m}$ thickness, and stained with H\&E solution (Sigma-Aldrich) to estimate inflammation.
Statistical analysis. Data are expressed as the means \pm the standard error of the mean (SEM). Statistical significance was determined using analyses of variance (ANOVAs) followed by multiple comparison tests with Dunnet's adjustment. $\mathrm{P}<0.05$ was considered significant.

\section{Results}

In vitro study

Effect of CL on cell viability. We determined the effect of CL on cell viability by MTT assay after incubating cells for $24 \mathrm{~h}$. The cytotoxicity of CL was preliminarily evaluated to establish the appropriate concentration ranges of CL for the analysis of ongoing experiments. Results showed that the cells' viabilities were not affected by $\mathrm{CL}$ at the concentrations $(5,10$, 20 and $40 \mu \mathrm{g} / \mathrm{ml}$ ) used (Fig. 1A). Therefore, we used non-toxic concentrations $(5-40 \mu \mathrm{g} / \mathrm{ml})$ for the entire experiment.

Effect of CL on the production of nitrite, $P G E_{2}$ and pro-inflammatory cytokines on LPS-stimulated RAW 264.7 cells. LPS-stimulated cells markedly increased in NO, whereas CL treatment significantly decreased NO production in a concentration-dependent manner (Fig. 1B). We also examined the effects of CL on $\mathrm{PGE}_{2}$ production following LPS stimulation in RAW 264.7 cells. The amount of $\mathrm{PGE}_{2}$ was increased by the LPS stimulation in the culture supernatant, and this increase was effectively reduced by treatment with CL (Fig. 1C).

Similarly, treatment of the RAW 264.7 cells with LPS alone resulted in a significant increase in cytokine production compared with the control group (Fig. 1D). However, CL treatment considerably inhibited the LPS induction of IL-6 in a dose-dependent manner (Fig. 1D).

Effect of CL on mRNA and protein expression levels of inflammatory mediators. The production of mRNA and protein of iNOS, COX-2 and pro-inflammatory cytokines, including IL-6 and TNF- $\alpha$, increased in LPS-stimulated RAW 264.7 cells (Fig. 2). However, treatment of the cells with CL significantly decreased iNOS, COX-2 and pro-inflammatory cytokine production compared to the LPS-stimulated cells in a concentration-dependent manner (Fig. 2).

Effect of CL on LPS-inducedMAPKactivation.LPS-stimulated cells showed an increase in the phosphorylation levels of p38, ERK1/2 and JNK. By contrast, treatment of the cells with CL significantly reduced the phosphorylation of p38, ERK and JNK expression compared with the LPS-stimulated cells in a concentration-dependent manner (Fig. 3).

Effect of CL on LPS-induced NF- $\kappa B$ activity. We examined the effect of CL on LPS-stimulated I $\kappa \mathrm{B}-\alpha$ degradation. One hour of pre-treatment with CL followed by treatment with LPS for 30 min markedly suppressed the LPS-stimulated phosphorylation and degradation of $\mathrm{I} \kappa \mathrm{B}-\alpha$ in a dose-dependent manner (Fig. 4A). We also investigated the translocation of the NF- $\kappa \mathrm{B}$ subunit p65 from the cytosol to the nucleus using western blot analysis. LPS stimulation caused p65 translocation from the cytosol to the nucleus, while CL inhibited this translocation (Fig. 4B). 

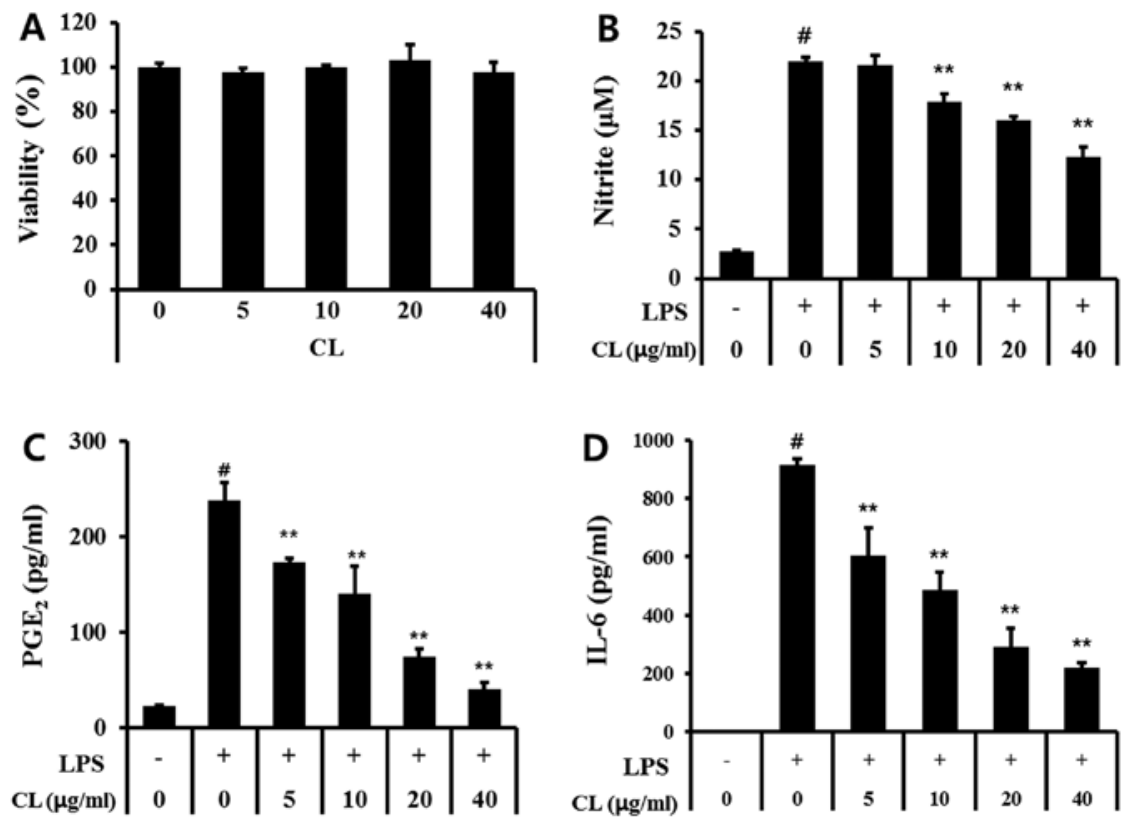

Figure 1. (A) Cytotoxic effects of Canarium lyi C.D. Dai \& Yakovlev (CL) in RAW 264.7 cells and the effects of CL on lipopolysaccharide (LPS)-induced nitric oxide (NO) (B), (C) prostaglandin E2 ( $\mathrm{PGE}_{2}$ ) and (D) interleukin-6 (IL-6) productions of RAW 264.7 macrophages. Cells were incubated for $24 \mathrm{~h}$ with $0.5 \mu \mathrm{g} / \mathrm{ml}$ of LPS in the absence or presence of CL $(0,5,10,20$ and $40 \mu \mathrm{g} / \mathrm{ml})$. CL was added $1 \mathrm{~h}$ before incubation with LPS. Cell viability assay was performed by using MTT assay. Nitrite concentration in the medium was determined by using Griess reagent. IL- 6 and PGE $_{2}$ levels in the medium were determined using

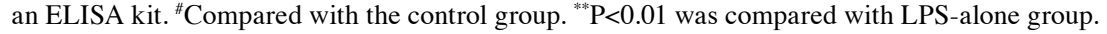
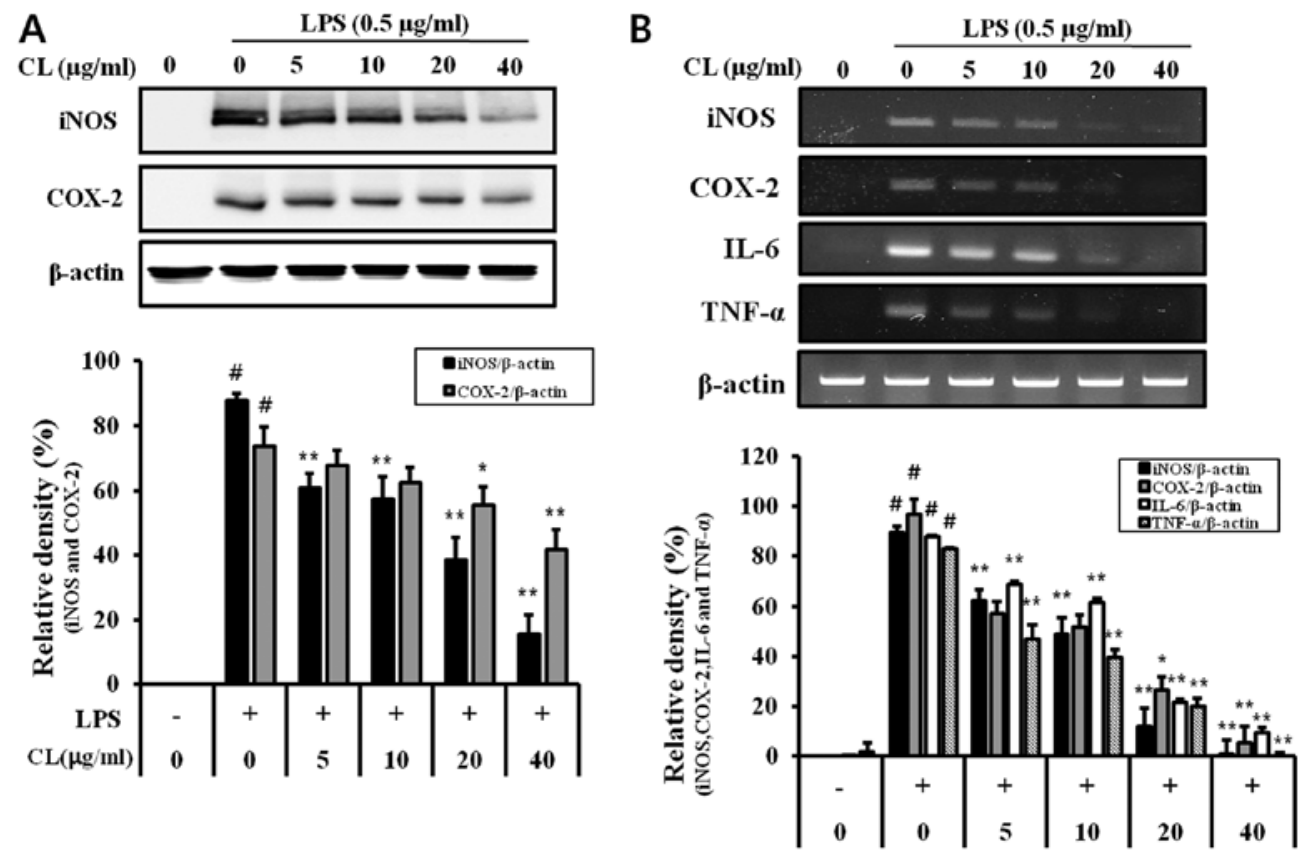

Figure 2. Effect of Canarium lyi C.D. Dai \& Yakovlev (CL) on mRNA and protein expression levels of inflammatory mediators in RAW 264.7 cells. Cells were pretreated with CL at various concentrations $(5,10,20$ and $40 \mu \mathrm{g} / \mathrm{ml}$ ) and then treated with lipopolysaccharide (LPS) for $18 \mathrm{~h}$. (A and B) Protein and mRNA expression levels of inducible nitric oxide synthase (iNOS) and cyclooxygenase-2 (COX-2) were determined by western blot analysis and RT-PCR. The mRNA expression levels of interleukin-6 (IL-6) and tumor necrosis factor- $\alpha$ (TNF- $\alpha$ ) were measured by (B) RT-PCR. " ${ }^{~ C o m p a r e d ~ w i t h ~ t h e ~ c o n t r o l ~ g r o u p . ~}{ }^{*}<<0.05$ and ${ }^{* *} \mathrm{P}<0.01$ were compared with the LPS-alone group.

\section{In vivo experiment}

Effect of CL on inflammatory cell count in the BALF of LPS-induced ALI mice. The LPS group showed a significant increase in the number of total inflammatory cells and neutrophils in BALF compared with the negative control group. However, pre-treatment with CL significantly decreased the number of total inflammatory cells and neutrophils compared with the LPS group (Fig. 5).

Effect of CL on pro-inflammatory cytokines in the BALF of $L P S$-induced ALI mice. The levels of IL-6, IL- $1 \beta$ and TNF- $\alpha$ in BALF were significantly higher in LPS-induced mice when 

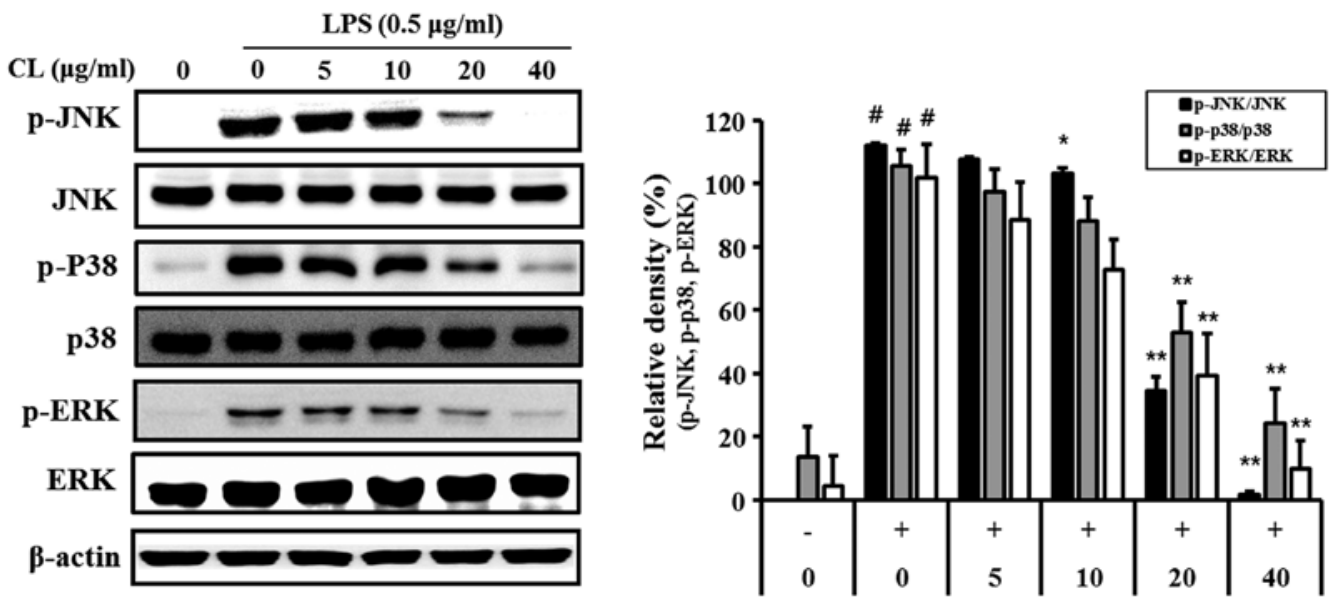

Figure 3. Effect of Canarium lyi C.D. Dai \& Yakovlev (CL) on lipopolysaccharide (LPS)-induced phosphorylation of p38 MAPK, extracellular signalregulated kinase (ERK) and c-Jun terminal kinase (JNK) in RAW 264.7 cells. We treated RAW 264.7 cells with various concentrations of CL (5, 10, 20 and $40 \mu \mathrm{g} / \mathrm{ml})$ for $1 \mathrm{~h}$ before stimulating the cells with LPS $(0.5 \mu \mathrm{g} / \mathrm{ml})$ for $30 \mathrm{~min}$. Cell extracts were subjected to western blotting with antibodies specific for the phosphorylation forms of $\mathrm{p} 38$, ERK, and JNK. ${ }^{\#}$ Compared with the control group. ${ }^{*} \mathrm{P}<0.05$ and ${ }^{* *} \mathrm{P}<0.01$ were compared with the LPS-alone group.

A

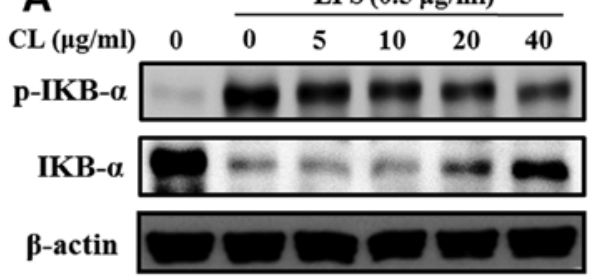

B

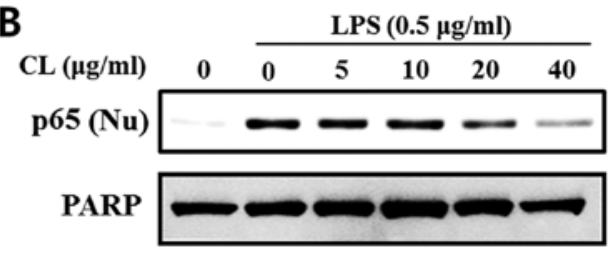

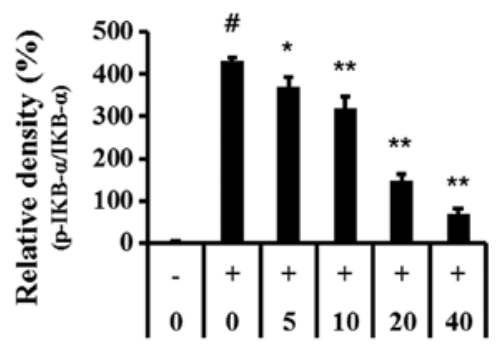

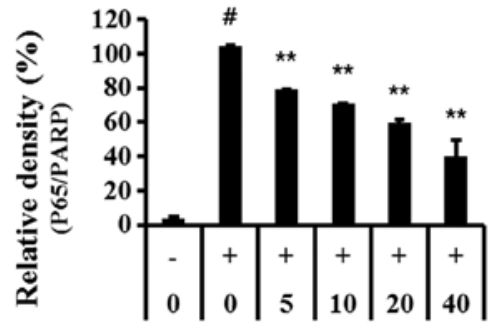

Figure 4. Effect of Canarium lyi C.D. Dai \& Yakovlev (CL) on lipopolysaccharide (LPS) induced the phosphorylation of I $\kappa-\alpha$ and nuclear factor- $\kappa$ B (NF- $\kappa$ B) nuclear translocation. Cells were incubated with various concentration of CL for $1 \mathrm{~h}$, and then stimulated with LPS $(0.5 \mu \mathrm{g} / \mathrm{ml})$ for $30 \mathrm{~min}$. Cytosol and nuclear extracts were assessed by western blotting. ${ }^{\# C}$ Compared with the control group. ${ }^{*} \mathrm{P}<0.05$ and ${ }^{* *} \mathrm{P}<0.01$ were compared with the LPS-alone group.

compared with the negative control group. By contrast, the CL-treated mice had considerably lower levels of all these pro-inflammatory cytokines compared to the LPS-treated mice (Fig. 6).

Effect of CL on COX-2 and NF- $\kappa B$ in the lung tissue of $L P S$-induced ALI mice. The LPS-induced mice exhibited an increased expression of COX-2, p-IкB and p-p65 in the lung tissue compared to the negative control group. However, treatment of the mice with CL significantly inhibited the expression of COX-2, I $\mathrm{B}$ degradation and the phosphorylation of p65 in the lung tissue compared to the LPS-treated mice (Fig. 7).

Effect of CL on histopathological changes in the lung tissue of LPS-induced ALI mice. We confirmed an intact structure and clear pulmonary alveoli in the lung sections of mice in the negative control group. By contrast, lung sections obtained from mice in the LPS group showed evidence of histological changes, including areas of inflammatory cell infiltration, thickening of the alveolar wall, edema and pulmonary congestion. By contrast, treatment of the mice with CL attenuated the pathological changes that were observed in the LPS-treated mice (Fig. 8).

\section{Discussion}

Macrophages are major inflammatory cells and immune effector cells (15). The activity of macrophages plays an important role in the inflammatory responses when infected with pathogens such as LPS.

Macrophages can kill pathogens directly by phagocytosis and indirectly through the secretion of various pro-inflammatory mediators such as reactive oxygen and pro-inflammatory cytokines such as TNF- $\alpha$, IL-1 $\beta$ and IL-6 (16). The overproduction 


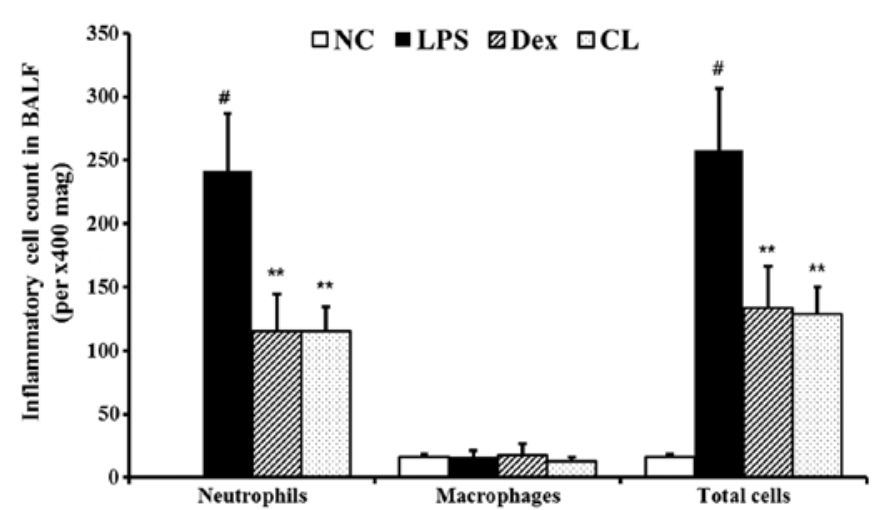

Figure 5. Effect of Canarium lyi C.D. Dai \& Yakovlev (CL) on the number of total cells, macrophages, and neutrophils in the bronchoalveolar lavage fluids (BALF) of lipopolysaccharide (LPS)-treated acute lung injury (ALI) mice. The mice of control and LPS groups received by oral gavage an equal volume of PBS. Mice included in the LPS, LPS + dexamethasone (DEX) and LPS + CL groups were instilled $10 \mu \mathrm{g}$ LPS dissolved in $50 \mu 1$ phosphate-buffered saline (PBS) intranasally to induce acute lung injury $1 \mathrm{~h}$ after final drug treatment. Mice in the control group were intranasally given $50 \mu 1$ PBS without LPS. We collected BALF at $18 \mathrm{~h}$ after the LPS challenge to measure the number of total cells, macrophages and neutrophils. ${ }^{~}$ Compared with the control group. ${ }^{* *} \mathrm{P}<0.01$ was compared with the LPS-alone group.
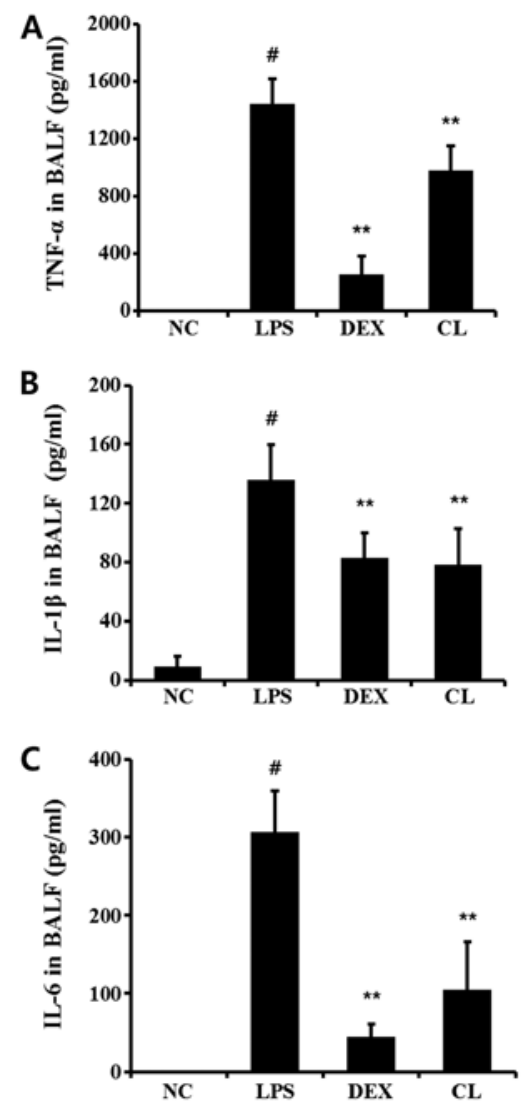

Figure 6. Effect of Canarium lyi C.D. Dai \& Yakovlev (CL) on the production of tumor necrosis factor- $\alpha$ (TNF- $\alpha$ ), interleukin-1 $\beta$ (IL-1 $\beta$ ) and interleukin-6 (IL-6) in the bronchoalveolar lavage fluids (BALF) of lipopolysaccharide (LPS)-treated acute lung injury (ALI) mice. The mice of control and LPS groups received by oral gavage an equal volume of phosphate-buffered saline (PBS). Mice included in the LPS, LPS + dexamethasone (DEX) and LPS + CL groups were administered $10 \mu \mathrm{g}$ LPS dissolved in $50 \mu \mathrm{l}$ PBS intranasally to induce acute lung injury $1 \mathrm{~h}$ after final drug treatment. Mice in the control group were intranasally given $50 \mu \mathrm{l}$ PBS without LPS. Bronchoalveolar lavage fluids (BALF) was collected $18 \mathrm{~h}$ following LPS. The inflammatory cytokines (A) $(\mathrm{TNF}-\alpha)$, (B) IL-1 $\beta$ and (C) (IL-6) were analyzed. " Compared with the control group. ${ }^{* *} \mathrm{P}<0.01$ was compared with the LPS-alone group.
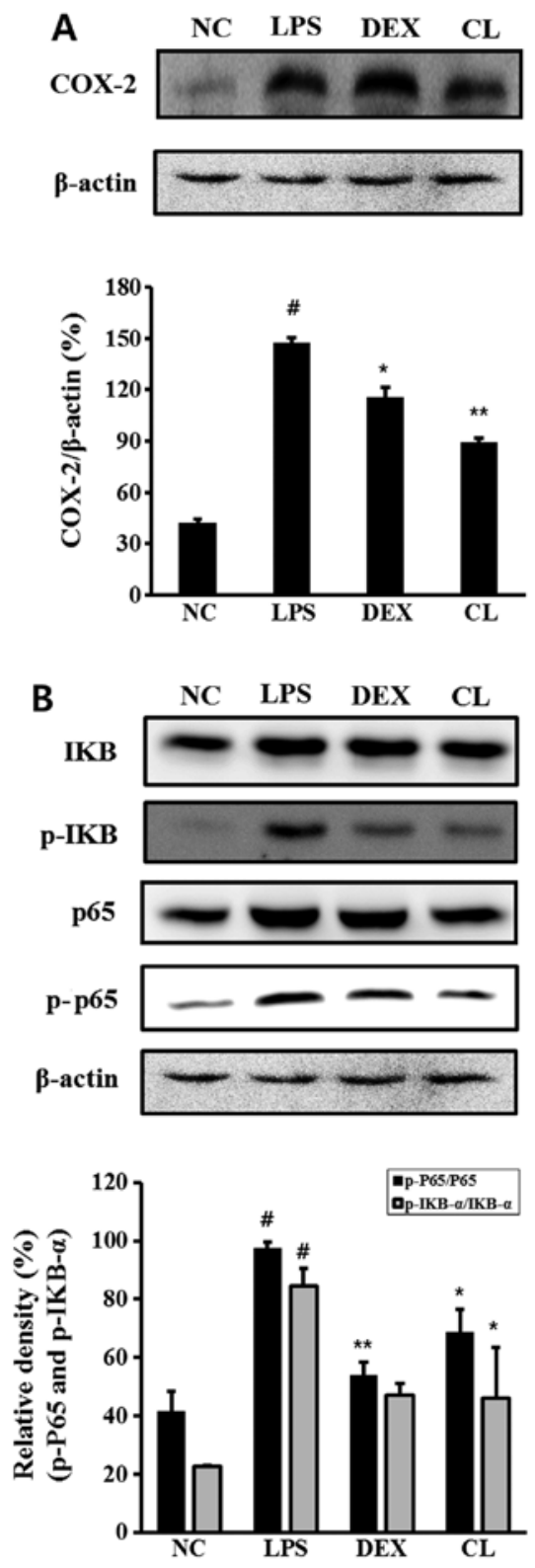

Figure 7. Effect of Canarium lyi C.D. Dai \& Yakovlev (CL) on cyclooxygenase-2 (COX-2), IкB and p65 in the lung tissue of lipopolysaccharide (LPS)-treated acute lung injury (ALI) mice. Mice of the control and LPS groups received by oral gavage an equal volume of phosphate-buffered saline (PBS). The mice included in the LPS, LPS + dexamethasone (DEX) and LPS + CL groups were administered $10 \mu \mathrm{g}$ LPS dissolved in $50 \mu 1$ PBS intranasally to induce acute lung injury $1 \mathrm{~h}$ after final drug treatment. Mice in the control group were intranasally given $50 \mu 1 \mathrm{PBS}$ without LPS. Lung tissues were collected $18 \mathrm{~h}$ following LPS. The protein expression of (A) cyclooxygenase-2 (COX-2), (B) IкB and p65 was analyzed. ${ }^{\#}$ Compared with the control group. ${ }^{*} \mathrm{P}<0.05$ and ${ }^{* *} \mathrm{P}<0.01$ were compared with the LPS-alone group.

of the inflammatory mediators by activated macrophages is involved in the pathophysiology of many inflammatory diseases, including arthritis, acute lung injury, chronic obstructive pulmonary disease (COPD), asthma and inflammatory bowel disease (IBD) (17). LPS can directly activate macrophages and trigger the production of inflammatory mediators, such as pro-inflammatory cytokines, $\mathrm{NO}$ and $\mathrm{PGE}_{2}$, which are the main cytotoxic and pro-apoptotic mechanisms participating in the innate response of many mammals. Thus, LPS-stimulated 


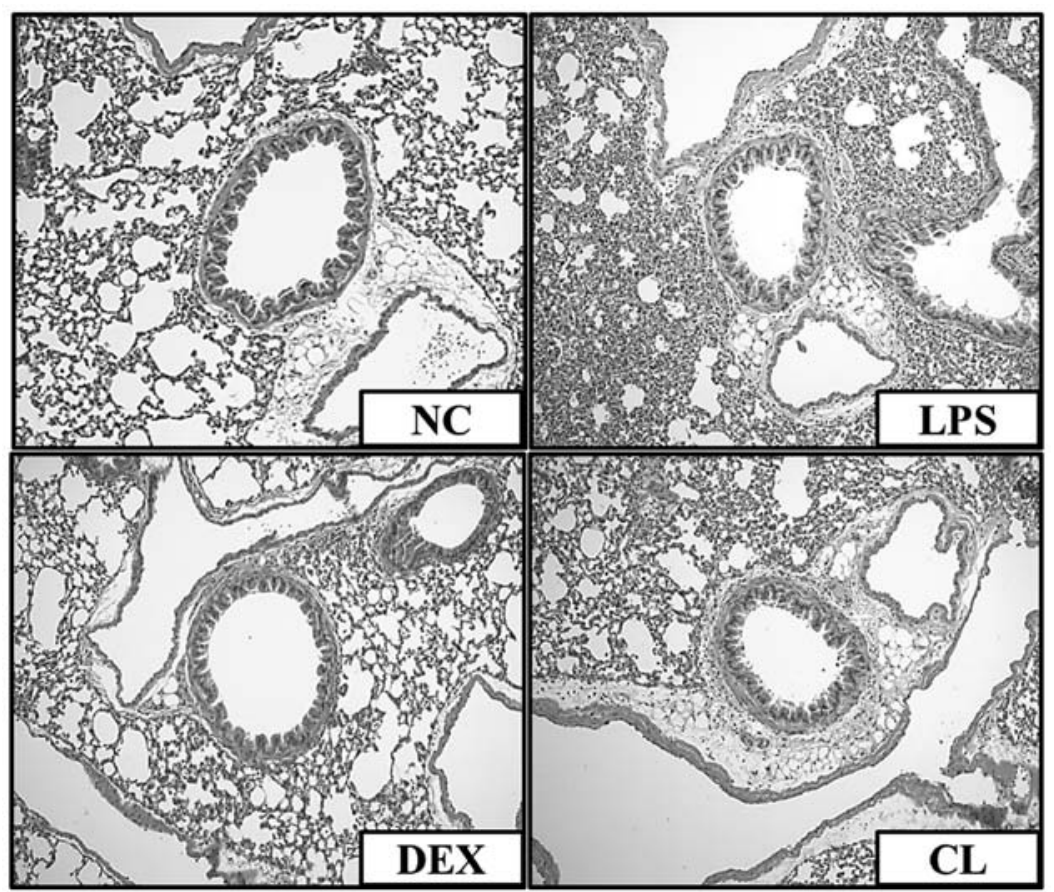

Figure 8. Effect of Canarium lyi C.D. Dai \& Yakovlev (CL) on histopathological changes in the lung tissue of lipopolysaccharide (LPS)-treated acute lung injury (ALI) mice. The mice received CL $(30 \mathrm{mg} / \mathrm{ml})$ by oral gavage $1 \mathrm{~h}$ before LPS intranasal instillation. Lungs from each experimental group were processed for histological evaluation $18 \mathrm{~h}$ after LPS intranasal instillation. Pathological changes of lung tissues were determined using hematoxylin and eosin staining.

RAW 264.7 macrophages can be effectively used as a model to study inflammation and potential anti-inflammatory mediators with their action mechanisms (18). The LPS stimulation of murine macrophages has been known to induce the phosphorylation and activation of MAPKs such as ERK 1/2, JNK, and p38 (19,20). Previous studies have demonstrated that LPS stimulation accelerates the phosphorylation of MAPKs in an inflammatory response. Moreover, it has been reported that the activation of NF- $\mathrm{NB}$ is triggered by MAPK (21). In the present study, we found that CL inhibited the expression of iNOS, COX-2, IL-6 and TNF- $\alpha$ mRNA simultaneously and concentration-dependently, suggesting that the inhibition of $\mathrm{NO}$ and $\mathrm{PGE}_{2}$ release may be caused by the suppression of iNOS and COX-2 expression at the mRNA level. In addition, we investigated the effects of CL on the LPS-stimulated phosphorylation of MAPKs in RAW 264.7 cells. The results showed that CL significantly reduced the phosphorylation of MAPKs in the LPS-stimulated RAW 264.7 cells compared with LPS-stimulated RAW 264.7 cells not treated with CL. Therefore, these results indicate that CL may attenuate the inflammatory responses induced by LPS stimulation.

$\mathrm{NF}-\kappa \mathrm{B}$ plays a central role in immunity since it activates the pro-inflammatory genes that encode iNOS, COX-2 and TNF- $\alpha$ (22). NF- $\kappa \mathrm{B}$ is a transcription factor and binds to the $\kappa \mathrm{B}$ motifs in the promoters of target genes, and thus, induces the transcriptions of iNOS, COX-2 and TNF- $\alpha$. In unstimulated cells, Rel protein dimers, which are composed mainly of p50 and p65 subunits, are sequestered in the cytoplasm as complexes with a family of inhibitors known as I $\kappa$ B (23). When the I $\kappa$ Bs become phosphorylated, NF- $\kappa$ B is released from its inhibition by $I \kappa B$ and translocated to the nucleus where inflammation-associated genes are then activated. $\mathrm{NF}-\kappa \mathrm{B}$ activation mediates the transactivation of pro-inflammatory genes, including TNF- $\alpha$ and IL-6 $(24,25)$. To elucidate the effect of CL on NF- $\kappa$ B activation, we evaluated the expression of phosphorylated $\mathrm{I} \kappa \mathrm{B}-\alpha$ in vitro and in vivo. The results of the present study demonstrate that $\mathrm{CL}$ blocked LPS-induced $\mathrm{I} \kappa \mathrm{B}-\alpha$ phosphorylation and, as a result, inhibited NF- $\kappa$ B activation. Since LPS induces changes in the $\mathrm{NF}-\kappa \mathrm{B}$ and MAPK signaling pathways, MAPKs may be another factor affected by CL exposure. These collective results provide convincing evidence that $\mathrm{CL}$ had an antiinflammatory ability by inhibiting LPS-stimulated NF- $\kappa$ B and MAPK activation and subsequent cytokine production in RAW 264.7 cells.

To investigate the potential anti-inflammatory effects of CL in vivo, we evaluated the protective effects of CL on a murine model of LPS-induced ALI. ALI is characterized by interstitial edema, neutrophil accumulation, epithelial integrity disruption, and protein leakage into the alveolar space, severely altering gas exchange (26). Many sequela associated with ALI result from the excessive production of cytokine mediators such as TNF- $\alpha$, IL-1 $\beta$ and IL-6 (27). Previous studies have shown that increased levels of TNF- $\alpha$, IL-1 $\beta$ and IL- 6 in BALF may be noted in the persistent elevation of pro-inflammatory cytokines in patients with ALI (28). TNF- $\alpha$, IL-1 $\beta$ and IL-6 increase the inflammatory cascade, cause inflammatory injury and recruit neutrophils into the lung (29). In our results, CL markedly decreased the production of TNF- $\alpha$, IL- $1 \beta$ and IL-6, as well as the infiltration of inflammation cells, including macrophages and neutrophils compared with LPS-induced ALI. These findings were consistent with the histopathology of lung tissue. These results indicate that the protective effects of CL on ALI induced by LPS may result from the inhibition of inflammatory mediators and the limitation of an inflammatory response in the lung. 
In summary, the results of the present study have demonstrated the anti-inflammatory effects of CL in in vitro and in vivo experiments. CL inhibited the expression of proinflammatory mediators in LPS-stimulated RAW 264.7 cells and LPS-induced ALI mice, and it blocked the activation of MAPKs and NF-kB. These results suggest that CL may have therapeutic potential for effectively treating inflammatory diseases such as pneumonia.

\section{Acknowledgements}

The present study was supported by a grant from the Ministry of Science, ICT and Future planning (FGC 1011332) and the KRIBB Research Initiative Program (KGM1221413) of the Republic of Korea.

\section{References}

1. Kim JJ, Jiang J, Shim DW, et al: Anti-inflammatory and antiallergic effects of Agrimonia pilosa Ledeb extract on murine cell lines and OVA-induced airway inflammation. J Ethnopharmacol 140: 213-221, 2012.

2. Huang MH, Wang BS, Chiu CS, et al: Antioxidant, antinociceptive, and anti-inflammatory activities of Xanthii Fructus extract J Ethnopharmacol 135: 545-552, 2011.

3. Hong YH, Chao WW, Chen ML, et al: Ethyl acetate extracts of alfalfa (Medicago sativa L.) sprouts inhibit lipopolysaccharideinduced inflammation in vitro and in vivo. J Biomed Sci 14: 16, 64, 2009.

4. Su YW, Chiou WF, Chao SH, et al: Ligustilide prevents LPS-induced iNOS expression in RAW 264.7 macrophages by preventing ROS production and down-regulating the MAPK, NF- $\kappa$ B and AP-1 signaling pathways. Int Immunopharmacol 11: 1166-1172, 2011.

5. Oh JH, Kang LL, Ban JO, et al: Anti-inflammatory effect of 4-O-methylhonokiol, compound isolated from Magnolia officinalis through inhibition of NF-kappaB. Chem Biol Interact 180 506-514, 2009.

6. Chen ZJ: Ubiquitin signalling in the NF-kappaB pathway. Nat Cell Biol 7: 758-765, 2005.

7. Park HH, Kim MJ, Li Y, et al: Britanin suppresses LPS-induced nitric oxide, PGE2 and cytokine production via NF- $\kappa \mathrm{B}$ and MAPK inactivation in RAW 264.7 cells. Int Immunopharmacol 15: 296-302, 2013.

8. Gao Y, Jiang W, Dong C, et al: Anti-inflammatory effects of sophocarpine in LPS-induced RAW 264.7 cells via NF- $\mathrm{kB}$ and MAPKs signaling pathways. Toxicol In Vitro 26: 1-6, 2012.

9. Dong C, Davis RJ and Flavell RA: MAP kinases in the immune response. Annu Rev Immunol 20: 55-72, 2002.

10. Kim JY, Shin JS, Ryu JH, et al: Anti-inflammatory effect of anemarsaponin B isolated from the rhizomes of Anemarrhena asphodeloides in LPS-induced RAW 264.7 macrophages is mediated by negative regulation of the nuclear factor- $\kappa \mathrm{B}$ and $\mathrm{p} 38$ pathways. Food Chem Toxicol 47: 1610-1617, 2009.

11. Saccani S, Pantano S and Natoli G: p38-dependent marking of inflammatory genes for increased NF-kappaB recruitment. Nat Immunol 3: 69-75, 2002.

12. Craig R, Larkin A, Mingo AM, et al: p38 MAPK and NF-kappa B collaborate to induce interleukin-6 gene expression and release. Evidence for a cytoprotective autocrine signaling pathway in a cardiac myocyte model system. J Biol Chem 275: 23814-23824, 2000 .
13. Huo M, Cui X, Xue J, et al: Anti-inflammatory effects of linalool in RAW 264.7 macrophages and lipopolysaccharide-induced lung injury model. J Surg Res 180: e47-e54, 2013.

14. Su YW, Chao SH, Lee MH, et al: Inhibitory effects of citronellol and geraniol on nitric oxide and prostaglandin $\mathrm{E}_{2}$ production in macrophages. Planta Med 76: 1666-1671, 2010.

15. Kim AR, Lee MS, Shin TS, et al: Phlorofucofuroeckol A inhibits the LPS-stimulated iNOS and COX-2 expressions in macrophages via inhibition of NF- $\mathrm{B}, \mathrm{Akt}$, and p38 MAPK. Toxicol In Vitro 25: 1789-1795, 2011.

16. Himaya SW, Ryu B, Qian ZJ, et al: Sea cucumber, Stichopus japonicus ethyl acetate fraction modulates the lipopolysaccharide induced iNOS and COX-2 via MAPK signaling pathway in murine macrophages. Environ Toxicol Pharmacol 30: 68-75, 2010.

17. Vodovotz Y, Chow CC, Bartels J, et al: In silico models of acute inflammation in animals. Shock 26: 235-244, 2006.

18. Huang SS, Chiu CS, Lin TH, et al: Antioxidant and antiinflammatory activities of aqueous extract of Centipeda minima. J Ethnopharmacol 147: 395-405, 2013.

19. Fengyang L, Yunhe F, Bo L, et al: Stevioside suppressed inflammatory cytokine secretion by downregulation of NF- $\kappa \mathrm{B}$ and MAPK signaling pathways in LPS-stimulated RAW264.7 cells. Inflammation 35: 1669-1675, 2012.

20. Tuntipopipat S, Muangnoi C, Chingsuwanrote $\mathrm{P}$, et al: Anti-inflammatory activities of red curry paste extract on lipopolysaccharide-activated murine macrophage cell line. Nutrition 27: 479-487, 2011

21. Cho EJ, An HJ, Shin JS, et al: Roxatidine suppresses inflammatory responses via inhibition of NF- $\kappa$ B and $\mathrm{p} 38$ MAPK activation in LPS-induced RAW 264.7 macrophages. J Cell Biochem 112: 3648-3659, 2011

22. Yun KJ, Shin JS, Choi JH, et al: Quaternary alkaloid, pseudocoptisine isolated from tubers of Corydalis turtschaninovi inhibits LPS-induced nitric oxide, $\mathrm{PGE}_{2}$, and pro-inflammatory cytokines production via the down-regulation of NF-kappaB in RAW 264.7 murine macrophage cells. Int Immunopharmacol 9: 1323-1331, 2009

23. An HJ, Kim IT, Park HJ, et al: Tormentic acid, a triterpenoid saponin, isolated from Rosa rugosa, inhibited LPS-induced iNOS, COX-2, and TNF- $\alpha$ expression through inactivation of the nuclear factor- $\mathrm{kb}$ pathway in RAW 264.7 macrophages. Int Immunopharmacol 11: 504-510, 2011.

24. Liu H, Sidiropoulos P, Song G, et al: TNF-alpha gene expression in macrophages: regulation by NF-kappa B is independent of c-Jun or C/EBP beta. J Immunol 164: 4277-4285, 2000.

25. Yoshimura A: Signal transduction of inflammatory cytokines and tumor development. Cancer Sci 97: 439-447, 2006.

26. Zhang Y, Du Z, Zhou Q, et al: Remifentanil attenuates lipopolysaccharide-induced acute lung injury by downregulating the NF- $\kappa \mathrm{B}$ signaling pathway. Inflammation: Apr 20, 2014 (Epub ahead of print).

27. Ward PA: Role of complement, chemokines, and regulatory cytokines in acute lung injury. Ann NY Acad Sci 796: 104-112, 1996.

28. Minamino T and Komuro I: Regeneration of the endothelium as a novel therapeutic strategy for acute lung injury. J Clin Invest 116: 2316-2319, 2006.

29. Matthay MA and Zimmerman GA: Acute lung injury and the acute respiratory distress syndrome: four decades of inquiry into pathogenesis and rational management. Am J Respir Cell Mol Biol 33: 319-327, 2005. 